\title{
HIGH RESOLUTION IMAGING OF BORON DISTRIBUTION ON DIAMOND FILM USING ENERGY FILTERED TEM
}

\author{
A. Dimyati ${ }^{1 *}$, S. Purwanto ${ }^{1}$ and R. Iskandar ${ }^{2}$ \\ ${ }^{1}$ Center for Advanced Material Science and Technology-BATAN, INDONESIA \\ ${ }^{2}$ Center Facility for Electron Microscopy, RWTH Aachen, GERMANY
}

\begin{abstract}
The main difficulty in investigation of thin film systems is the lack of capability to get detail information of the material in nano level due to the low resolution of conventional imaging techniques such as SEM, SIMS etc. In this work Electron Spectroscopy Imaging (ESI) in energy filtered transmission electron microscope (EFTEM) was used to produce a real image of boron distribution in a diamond film deposited on (111) Si by chemical vapor deposition. The result revealed the layer consists of $1.3 \mu \mathrm{m}$ thick diamond structured carbon film adjacent to Si substrate and $120 \mathrm{~nm}$ amorph carbon layer on top most surface. Boron atoms were distributed uniformly in both layer, however slight higher concentration in the second layer is observed. There was obviously no grain boundary enrichment of Boron atoms observed.
\end{abstract}

Keywords: boron doped diamond film, CVD, ESI, EFTEM

*E-mail address: arbi_dimyati@hotmail.com

\section{Introduction}

Due to its superior properties Diamond film have many advantages in applications as field emitter material, micro mechanical devices and semi conducting material in many areas from household to nuclear technique [1-4]. Recently Purwanto et al. reported their observation of the magnetoresistance property of implanted diamond film which opens a new application in the field of sensor technology [5]. Today research works of this material focus more on the attempts to enhance those properties by incorporation of small elements such as boron, iron or nitrogen either by ion implantation or vapor deposition technique. The effect of boron on the property changes of diamond films is widely accepted to be strongly depending on its distribution and on the diamond film grain size. The typical size of diamond crystal grain ranges from view microns down to some nanometers. However in case of application as FEE material the nano or subnanometer size is much preferred [6].

Up to now microstructural investigations of diamond films have been mainly performed either by conventional scanning electron microscopy (SEM), Raman spectroscopy or secondary ion mass spectroscopy (SIMS). However the mean dimension of the regions in the material in which the electronic processes usually happen is in the order of nanometer or even sub-nanometer. Therefore imaging of materials in this size requires devices that exhibit true imaging and analytical capability at very high magnification.

SEM investigation can provide interpretable image at magnifications as high as 20.000 times with relative good contrast and sufficient focus. But it cannot resolve the matter of interest at higher magnification without loss of resolution. In this work a special technique for imaging of doping atom distribution the $\mathrm{Fe}+\mathrm{B}$ doped diamond film using energy filtered transmission electron microscopy (EFTEM) is presented with the aim to elucidate the fine structure of the films including boron distribution in order to get better insight into its correlation to the magnetoresistance property of the films. The SEI technique is discussed.

\section{Experimental Method}

CVD diamond film with $1.3 \mu \mathrm{m}$ thickness on Silicon wafer (111) was supplied by Applied Diamond Inc. USA. Two samples were doped by Fe$\mathrm{B}$ ion implantation with dose $10^{15}$ ions $/ \mathrm{cm}^{2}$ at 70 $\mathrm{keV}$ and followed by Argon ion sputtering at 70 $\mathrm{keV}$. For imaging of the boron atoms distribution Energy filtered TEM FEI Tecnai G2 $200 \mathrm{keV}$ equipped with Gatan post column Electron Energy Loss Spectroscopy (EELS) was used. The samples were prepared in the Focus Ion Beam workstation (FIB) FEI Strata 205 giving sufficiently large transparent area and subsequent plasma cleaning in $\mathrm{N}$ gas atmosphere to minimize the $\mathrm{Ga}$ ion bombardment induced defects. 


\subsection{ESI Imaging}

The basic set up of the EFTEM used in this work is shown in Fig. 1. The energy filtering is performed in the EELS spectrometer situated in post column position consisting mainly of a magnetic prism, energy selecting slit and a series of magnifying lenses. The magnetic prism extracts the electrons of different energy loss similar to process happens to white light in an optical prism as shown schematically in Fig. 2.

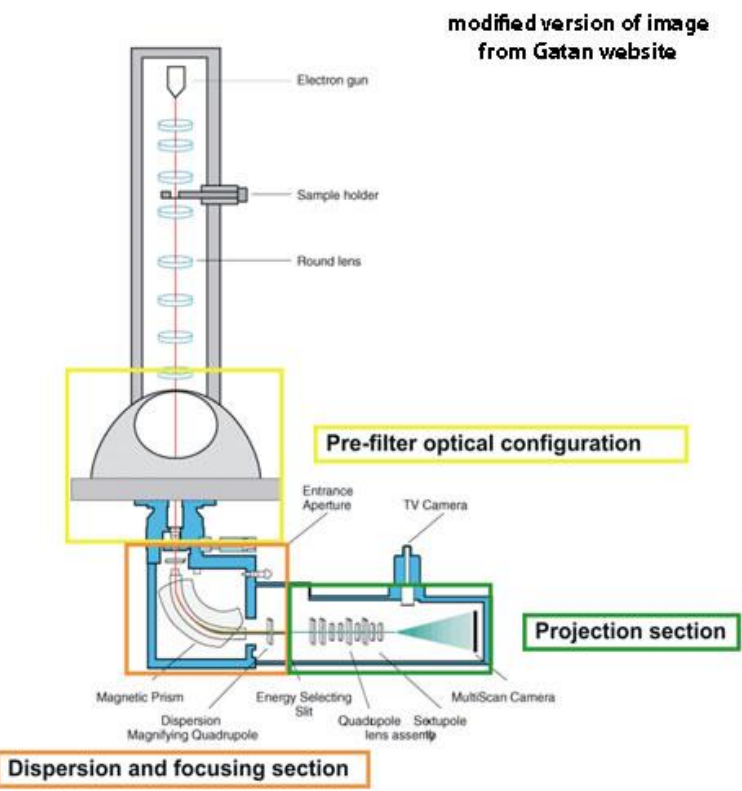

Fig.1. Block diagram of TEM with GATAN energy filtering

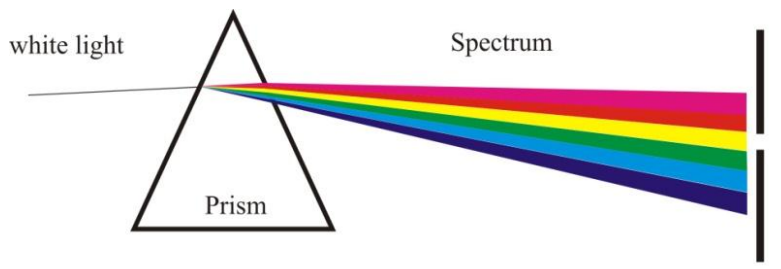

Selecting slit

Fig.2. White light diffraction in an optical prism. Mechanical slit selected an electron with certain energy loss for further analysis or imaging

The result of EELS measurement is an intensity spectrum of inelastically scattered electrons as a function their loss of energy due to interaction with the sample, as shown for example in Fig. 3. The spectrum contains characteristic edges of elements with them primary electron beam has been interacted. This is useful information for analysis or in connection with this work for imaging of element.

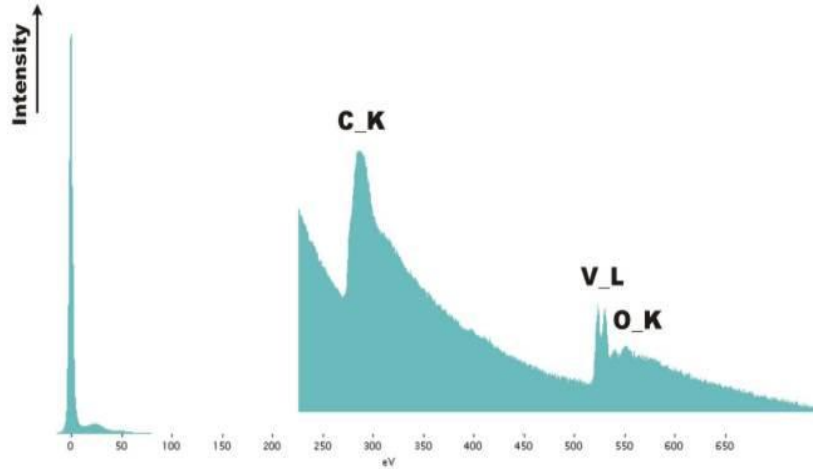

Fig.3. EELS spectrum showing characteristic edge peaks of electron with certain energy loss

For quantification the corresponding edge of element is calculated by simply subtraction the background intensity to its peak. However to take into account the background curve under the peak, three windows technique for calculation of the peak intensity is performed as shown in Fig. 4. Detail description of this subtraction technique can be found in [7].

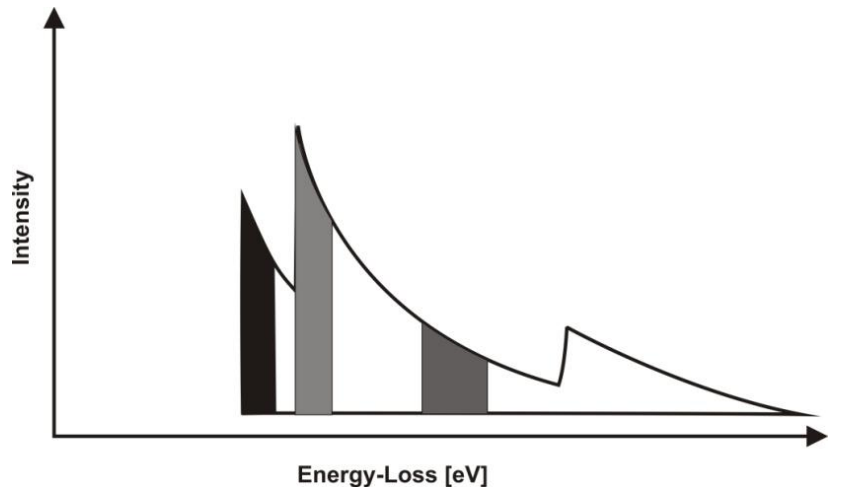

Fig.4. Three windows technique to quantify the edge

For imaging (two dimensional) of the distribution of element, a series of EELS spectrums from a selected area of interest are pixel by pixel acquired and calculated at the same time as schematically described in Fig. 5. 

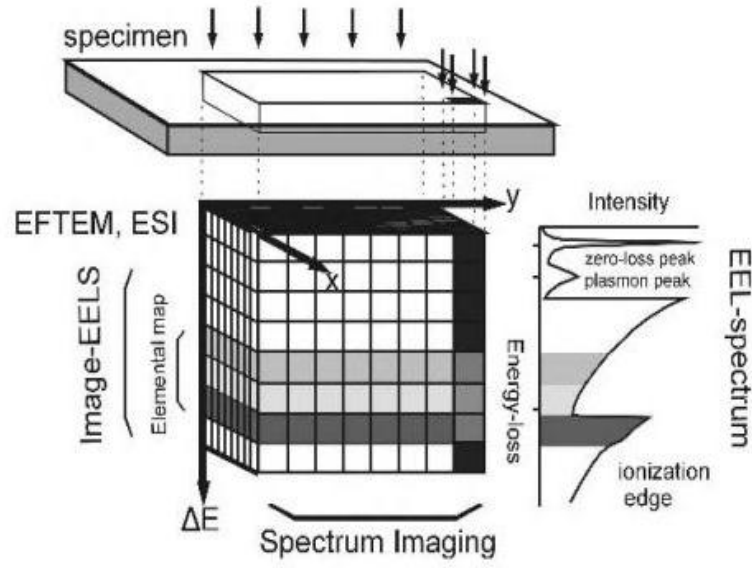

Fig.5. Principle of two imaging of element by ESI technique

\section{Results and Discussion}

Fig. 6 shows secondary electron (SE) image of the diamond film cross section taken by Field Emission Scanning Electron Microscopy (FESEM) tilted about $3^{\circ}$ against horizontal axis emphasizing the main characteristic microstructure of the film. One can observed, the layer surface is obviously not flat exhibiting characteristic pyramid like structure which is distributed inhomogenously throughout observed area. The measured thickness is in the range between 8667 to $1300 \mathrm{~nm}$.

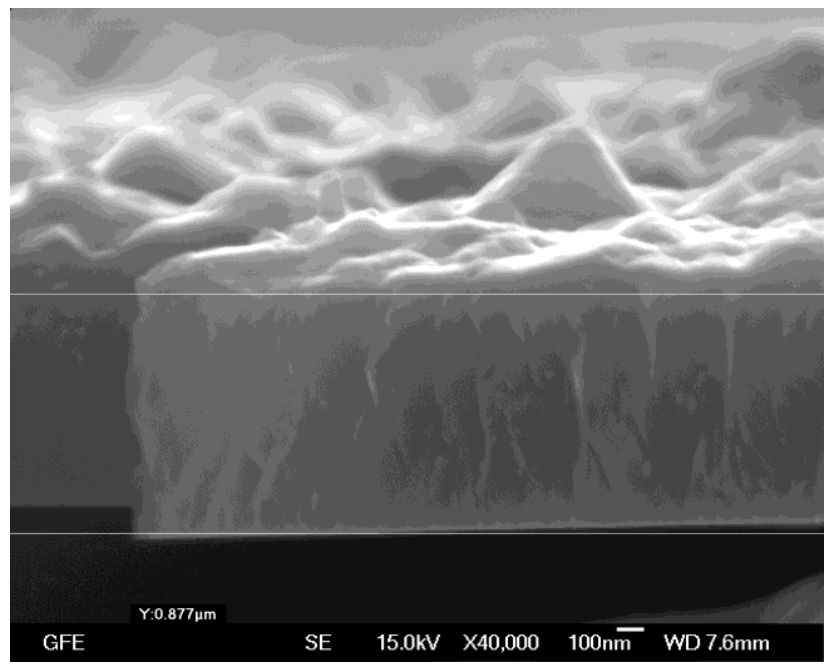

Fig.6. FESEM cross section of $\mathrm{Fe}+B$ implanted diamond film

The corresponding EFTEM brightfield image is given in Fig. 7 left presents the detail microstructure of diamond layer consisting of columnar grown diamond crystals adjacent to Si-matrix surface and about $100 \mathrm{~nm}$ thick amorphe carbon layer on top most surface. The image is indicated by extremely good contrast showing almost every single diamond crystal with clear observable grain boundaries. On the top of the sample surface is tungsten protective layer and appears dark. This layer is used to prevent any destruction of the sample surface by heavy $\mathrm{Ga}$ ion during FIB preparation. In other word the existence of tungsten layer indicates the originity of sample surface before and after FIB preparation.

The result of ESI imaging using Boron atom edge is presented in Figure 7 right. EELS spectra collection and calculation has taken less than 3 minutes. The distribution of Boron atoms is indicated by white contrast in the dark background. Dark means the absent of by Boron atoms. The Boron atoms seem to occupy the area of interest shown in corresponding EFTEM brightfield image relative homogenously in both of crystalline and amorphe region. However the concentration of Boron atoms in the amorphe layer is obviously higher than in the adjacent layer. Due to the sensitivity of EELS detection down to atomic level, every single bright pixel in the ESI image can be interpreted as coming from single or view Boron atoms.

As can been seen in the ESI image, Boron atoms are located only in the diamond layer. The Si-matrix surface is relatively free of Boron atoms.

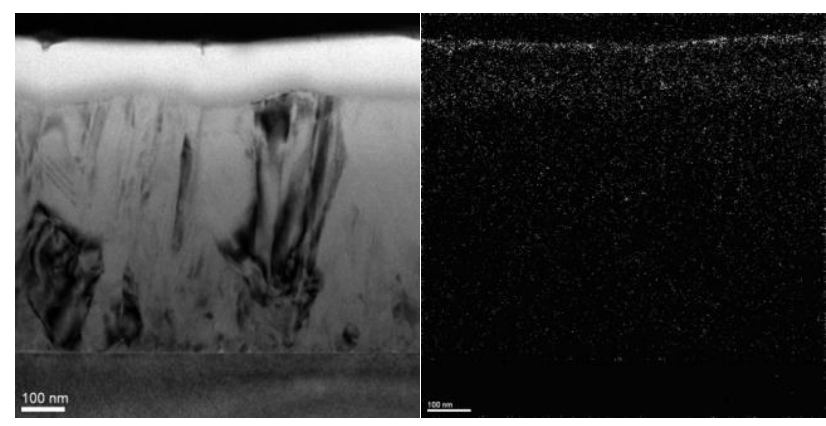

Fig.7. EFTEM image of $\mathrm{Fe}+\mathrm{B}$ implanted diamond film and the distribution of boron atom taken by ESI technique.

\section{Conclusion}

Imaging of Boron atom distribution with atomic resolution using electron spectroscopy imaging (ESI) has been successfully performed. Boron atoms have occupied the entire diamond layer. However the Boron concentration distributed in the crystalline layer was lower than those in the amorphe layer. ESI imaging technique is therefore one of promising technique to elemental map at higher.

\section{Acknowledgements}

The authors would like to thank to Prof. Mayer for fruitful discussion and for using the GFE facility 
during EFTEM measurement. The two of the authors thank to Ministry of Research and Technology of Republic Indonesia for financial support.

\section{References}

[1] Hampsel, F.J. Van Venchten, J.A. \& Eastmann, D.E. Phys. Rev. B 1979; 20: p. 624.

[2] Pate, B.B. Stefan, P.M. Binns, C. Jupiter, P.J. Shek, M.L. landau, I. \& Spicer, W.E. J. Vac. Sci. Technol. 1981; 19: p. 349.

[3] Kohn, E. Gluche, P. \& Adamschik, M. Diamond MEMS a new emerging technology. Diamond and Related Materials 1999;8 (2-5): p. 934-940.

[4] Tang, Y. X. \& Aslam, D. M. Technology of polycrystalline diamond thin films for microsystems applications. Journal of Vacuum Science \& Technology B23 2005; 3: p. 10881095.

[5] Purwanto, S. Iskandar, R. A. A Wisnu, Tjipto, S. \& Dimyati, A. accepted by Makara Journal.

[6] Pankaj, Koinkar, M. Sandip, Patil, S. Kim,T. G. Yonekura, D. \& Mahendra More, A. Dilip, Joag, S. \& Murakami, R. Applied Surface Science 2011; 257: p. 1854-1858.

[7] Pengkalla, H.-J. Conf. on Electron Microscopy of Solids, Poland 1999: p. 37-44. 